\title{
Topical antifungal bigels: Formulation, characterization and evaluation
}

\author{
AGNE MAZURKEVICIUTE ${ }^{1 *}$ \\ KRISTINA RAMANAUSKIENE ${ }^{1}$ \\ MARIJA IVASKIENE ${ }^{2}$ \\ AIDAS GRIGONIS $^{2}$ \\ VITALIS BRIEDIS \\ ${ }^{1}$ Department of Clinical Pharmacy \\ Faculty of Pharmacy, Lithuanian \\ University of Health Sciences, 50161 \\ Kaunas, Lithuania \\ ${ }^{2}$ Department of Non-infectious \\ Diseases, Faculty of Veterinary \\ Medicine, Veterinary Academy \\ Lithuanian University of Health \\ Science, 47181 Kaunas, Lithuania
}

Accepted December 21, 2017

Published online February 6, 2018

\begin{abstract}
Bigels with antifungal substances, ciclopirox olamine and terbinafine hydrochloride, were made of hydrogel (poloxamer 407 gel) and oleogel (polyethylene and liquid paraffin mixture). Prepared bigels were found physically stable at room temperature for six months and at least four months at $40^{\circ} \mathrm{C}$. Released amount of drug decreased when oleogel concentration in the formulation increased. Release test results depended on the insertion place of active substances. The amount of released substance was highest when ciclopirox olamine was incorporated in both phases in an equal quantity, and terbinafine hydrochloride in oleogel or in hydrogel. All formulations showed great inhibition of Microsporum canis. Thus, bigels with ciclopirox olamine and terbinafine hydrochloride are a promising dosage form for topical use.
\end{abstract}

Keywords: bigel, ciclopirox olamine, terbinafine hydrochloride, poloxamer 407, antifungal, Microsporum canis

Topical semi-solid formulations are used to treat various skin and systemic diseases. They help avoid hepatic first-pass metabolism and serum absorption is minimal; furthermore, high drug concentration in skin is achieved by topical administration (1). This is important for long-term therapy, for example, therapy of fungal diseases, which can be classified into superficial, subcutaneous and systemic mycoses. The most common fungal infections are dermatophytoses.

We hypothesized that incorporation of antifungal active substances in a gel and bigel could ensure suitable contact of the drug and the skin and its sufficient release. Bigel (oleogel/hydrogel, oleo-hydrogel, biphasic gels) is a semi-solid formulation that consists of two phases: hydrogel and oleogel (2). Gels are semi-solid matrices comprising small amounts of a solid dispersed in a relatively large amount of a liquid. Gels might provide faster drug release compared to creams and ointments, regardless of water solubility of the substance (3). Bigels are stable, non-oily systems that combine advantages of hydrogels and oleogels: ease of preparation, possible delivery of lipophilic and hydrophilic drugs, absence of surfactants (4). The oil phase helps moisturize the skin, prevents water loss and ensures uni-

\footnotetext{
*Correspondence; e-mail: agne.mazurkeviciute@lsmuni.lt
} 
form distribution of the drug substance on the skin. Bigels differ from emulgels because both phases are structured (5) while in emulgels the internal phase is a liquid.

Two widely used drugs were chosen as model substances: ciclopirox olamine (CPO) and terbinafine hydrochloride (TFH). Both compounds have broad antifungal activity against dermatophytes, yeasts and other nondermatophytes and are used to treat cutaneous and subcutaneous mycoses in humans and in veterinary medicine (6).

TFH is an allylamine, squalene epoxidase inhibitor (7). TFH is well tolerated and is also administered orally (1). Terbinafine hydrochloride is used in various topical forms: cream, gel, solution.

Ciclopirox is a hydroxyl-pyridone derivate. Ciclopirox reduces the activity of metal-dependent enzymes that affect ion transport through membranes (15). CPO has also a broad antibacterial and anti-inflammatory activity. Ciclopirox can be a free acid or ethanolamine salt. Ciclopirox olamine is used in lacquers, shampoos, solutions, suspensions, creams and gels (15). These substances are used in gel formulations, but there is no published research on bigel formulations. It is relevant to investigate the suitability of bigels to introduce the tested active substances and achieve effective therapeutic effects.

According to some scientific articles, bigels could be unstable at high temperatures because of phase separation (2). Hence, it is important to evaluate the influence of temperature on the formulation stability. The principal objective of this article is to evaluate the stability and quality of bigels prepared with different proportions of hydrogel and oleogel according to biopharmaceutical tests in vitro, antifungal activity assay, rheological tests.

\section{EXPERIMENTAL}

\section{Materials}

Ciclopirox olamine and terbinafine hydrochloride were supplied by Chemical Point (Germany). Hydroxypropyl cellulose, $M_{\mathrm{r}} 100.000$ was purchased from Alfa Aesar (Germany). Poloxamer 407 was purchased from Fagron (The Netherlands). Ethanol $>96 \%$ was from Spiritus Vilnensis (Lithuania). Oleogel Pionier PLW (mixture of liquid paraffin and polyethylene) was received from Hansen \& Rosenthal KG (Germany) as a gift sample.

\section{Preparation of bigels}

Different gels with CPO and TFH were formulated and evaluated in previous studies (9). The highest release was recorded from poloxamer 407 gels. Therefore, studies with poloxamer 407 are continued. Poloxamer 407 was mixed with distilled water and kept in refrigerator at $+4{ }^{\circ} \mathrm{C}$ for 12 hours. The required amount of terbinafine hydrochloride/ ciclopirox olamine was then dissolved in a cold poloxamer solution or mixed with oleogel. The cold solution was then allowed to warm up to room temperature and hydrogel was formed (9). Prepared hydrogel was then mixed with oleogel at room temperature until a homogenous formulation was obtained (2). Bigel compositions are given in Table I.

Bigels with different amounts of model substances in phases were prepared in order to estimate how that influenced the released amount of active substance. Four different types of each formulation were prepared: A - active substance in hydrogel, B - amount of active substance in the phase was proportional to the amount of the phase in formulation, 
$\mathrm{C}$ - equal amounts of active substance in both phases, D - active substance in oleogel. Amounts of TFH/CPO in oleogel and hydrogel are given in Table II.

Before the measurement of rheological properties, the bigels were kept at room temperature for 24 hours.

\section{Structure analysis}

The microstructure of bigels was investigated with the aid of an optical microscope analysis. Micrographs were obtained using a Motic microscope (Motic Instruments, Inc.) equipped with a 100× objective. Computer software Motic images were used.

\section{Rheological measurements}

Rheological measurements were performed with a model MCR102 rotational rheometer (Anton Paar, Austria) equipped with a plate-plate geometry (gap $1 \mathrm{~mm}$ ); temperature was controlled with a Peltier system. Rheological properties were assessed at $32{ }^{\circ} \mathrm{C}$. Flow curves were done in the range from 0.01 to $100 \mathrm{~s}^{-1}$. The power-law model was adapted. Flow behaviour index $n$ and consistency index $K$ were calculated. All tests were repeated three times and averaged results are shown.

\section{In vitro release test}

An in vitro release study was performed with natural cellulose membranes Cuprophan (Medicell International Ltd., UK), mounted in modified Franz type diffusion cells. The diffusion area was $1.77 \mathrm{~cm}^{2}$. An infinite dose of the donor phase was placed into the diffusion cell. The aqueous receptor medium was stirred using a hotplate magnetic stirrer IKAMAG C-MAG HS7 (IKA-Werke GmbH \& Co.KG, Germany) maintaining the temperature at $32{ }^{\circ} \mathrm{C}$. Samples of the receptor solution were collected at 1, 2, 3, 4, 5, 6 hours and replaced with the same volume of fresh receptor solution. All samples were analysed with a spectrophotometer (Agilent 8453, Australia) at the wavelength of maximum absorption of CPO (303 nm), TFH (283 nm).

\section{Antifungal activity study}

Tested bigels were studied for their in vitro antifungal activity against Microsporum canis using the agar well diffusion technique (9). Liquid sterile Sabouraud dextrose agar with chloramphenicol was used for the preparation of cultures and incubation of fungal species. M. canis was isolated from cats affected with dermatophytosis and from 7 to 14 day cultures were grown on Sabouraud dextrose agar. The prepared dermatophyte inoculi $\left(1.0 \times 10^{6}-5.0\right.$ $\times 10^{6}$ spores per $\mathrm{mL}$ ) were seeded over the surface of clotted SDA plates and allowed to dry. One well with a $7-\mathrm{mm}$ diameter was made in each plate. About $0.1 \mathrm{~mL}$ of the bigel was delivered into the well using a micropipette. The plates were incubated at $28^{\circ} \mathrm{C}$ for 7 days. Antifungal activity was evaluated by measuring the zone of inhibition in millimetres.

\section{Stability study}

The bigels were stored in climatic chambers over 6 months at $25^{\circ} \mathrm{C} / 60 \% \mathrm{RH}$ (relative humidity) and $40{ }^{\circ} \mathrm{C} / 75 \% \mathrm{RH}$ and were checked at regular intervals for physical instability. The rheological, microscopic and organoleptic features were observed. Formulations were no longer investigated after phase separation. 


\section{Statistical analysis}

All tests were repeated three times. The mean values and standard deviations of the results were calculated using IBM SPSS statistics 20 and Microsoft Office Excel 2016 programs. The significance of differences was evaluated using Student's $t$-test. The differences were statistically significant at $p<0.05$.

\section{RESULTS AND DISCUSSION}

\section{Structure analysis}

In the first part of this research, we evaluated the structure of formulations. Prepared bigels were of milky white colour. Micrographs (Fig. 1) showed the presence of droplets. The droplets became larger and of irregular shape as the concentration of oleogel in formulations increased. The size of the droplets of Bi-10, Bi-20, Bi-30 and Bi-40 was up to 38, 67,80 and $100 \mu \mathrm{m}$, respectively. Bigger droplet sizes in Bi-30 and Bi-40 might have resulted in phase separation during the accelerated stability study.
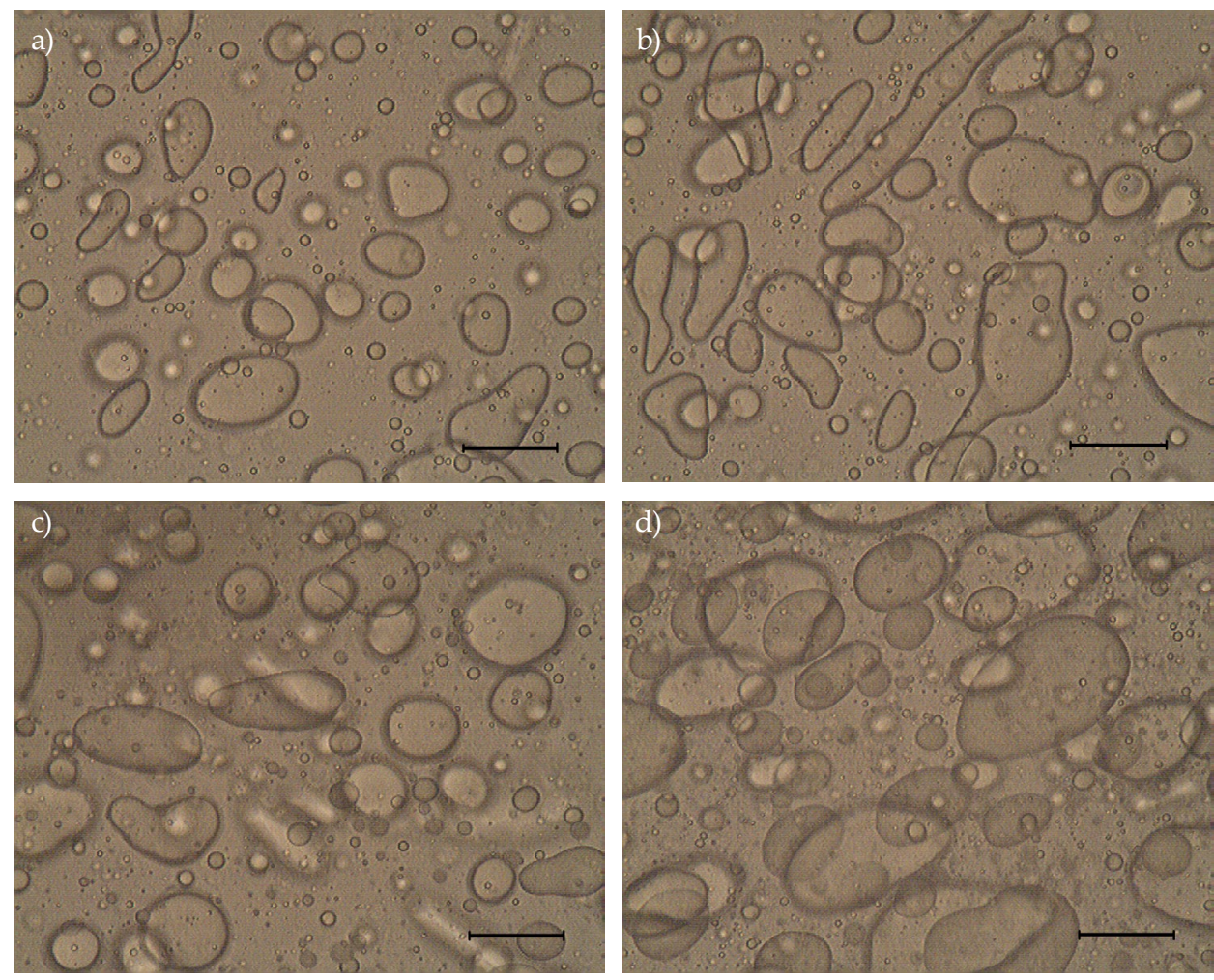

Fig. 1. Microstructure of bigels: A - Bi-10, B - Bi-20, C - Bi-30, D - Bi-40. Reference bar corresponds to $50 \mu \mathrm{m}$. 


\section{Rheological tests}

Rheological studies are relevant to quality evaluation of semi-solid preparations and allow identifying product viscosity and structure changes with temperature (10). Even if there are no visible changes, a rheological test indicates changes in the internal structure and predicts the stability of products. A flow curve can be used to determine whether the rheological behaviour of the formulation is Newtonian or non-Newtonian and indicates product quality. Power law or Ostwald de Waele model is the mathematical model that describes pseudoplastic or shear-thickening behaviour: $\tau=K \gamma^{n}$, where $\tau$ is shear stress (Pa), $K$ is consistency index $\left(\mathrm{Pa} \mathrm{s}^{\mathrm{n}}\right), \gamma$ is shear rate $\left(\mathrm{s}^{-1}\right), n$ is flow behaviour index (dimensionless). The consistency index is relevant for liquid consistency (10). The flow behaviour index represents the departure from Newtonian behaviour ( $n=1$ for Newtonian fluids) and is a measure of pseudoplastic $(n<1)$ or shear- thickening $(n>1)$ extent of the system (11).

Based on the study results, it can be stated that oleogel affects the structure of bigels. The flow curves are shown in Fig. 2. Values of the flow behaviour index were between 0.1 and 0.2. As a part of oleogel in formulation is increasing, the flow behaviour index increases (structures get weaker). There were statistically significant differences $(p<0.05)$ between the flow behaviour indexes of different formulations. Consistency index decreases when the amount of oleogel in formulation increases. This result might be due to the flow of the droplets.

\section{In vitro drug release}

In biopharmacy, products are evaluated by a release test in vitro. This study allows assessing the suitability of the base to relax the substance. The results depend on the nature of the drug and its solubility in the base, viscosity of the pharmaceutical form, and acceptor medium (12).

The analysis of release study results demonstrates that the release of drug from semisolid vehicles was influenced by various factors: type of substance, amount of oleogel in the formulation and drug incorporation in phases (Table III). The results confirmed that the excipients have an impact on the release of active substances (13). The released amount

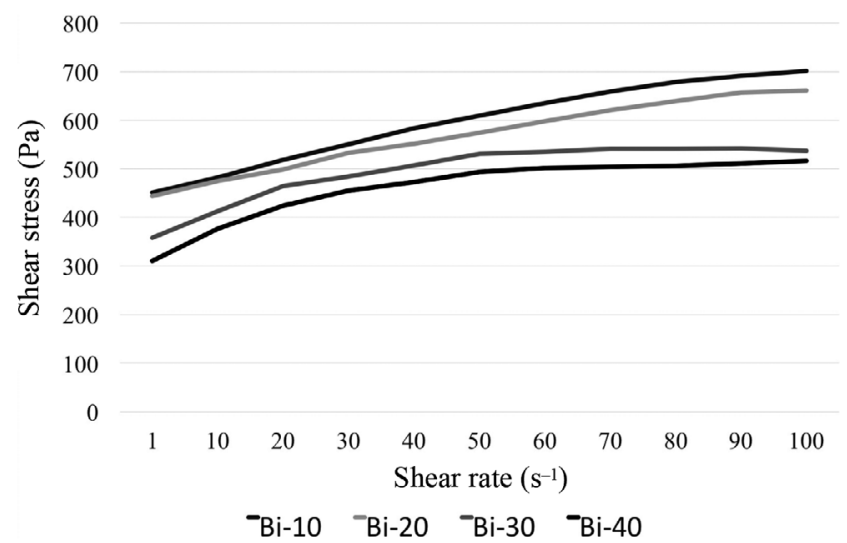

Fig. 2. Flow curves of bigels $(n=3)$. 
Table I. Compositions of different bigel samples

\begin{tabular}{cccccc}
\hline $\begin{array}{c}\text { Composition } \\
(\%, m / m)\end{array}$ & $\begin{array}{c}\text { Terbinafine } \\
\text { hydrochloride }\end{array}$ & $\begin{array}{c}\text { Ciclopirox } \\
\text { olamine }\end{array}$ & Water & $\begin{array}{c}\text { Poloxamer } \\
407\end{array}$ & Oleogel \\
\hline Bi-10 T & 1.0 & - & 66.8 & 22.3 & 9.9 \\
Bi-20 T & 1.0 & - & 59.3 & 19.8 & 19.9 \\
Bi-30 T & 1.0 & - & 51.8 & 17.3 & 29.9 \\
Bi-40 T & 1.0 & - & 44.3 & 14.8 & 39.9 \\
Bi-10 C & - & 1.0 & 66.8 & 22.3 & 9.9 \\
Bi-20 C & - & 1.0 & 59.3 & 19.8 & 19.9 \\
Bi-30 C & - & 1.0 & 51.8 & 17.3 & 29.9 \\
Bi-40 C & - & 1.0 & 44.3 & 14.8 & 39.9 \\
\hline
\end{tabular}

Table II. The amounts of TFH/CPO in different phases: oleogel and hydrogel $(\%, \mathrm{~m} / \mathrm{m})$

\begin{tabular}{cccccc}
\hline Formulation & & $\mathrm{A}$ & $\mathrm{B}$ & $\mathrm{C}$ & $\mathrm{D}$ \\
\hline \multirow{2}{*}{ Bi-10 } & hydrogel & 1.0 & 0.9 & 0.5 & 0.0 \\
& oleogel & 0.0 & 0.1 & 0.5 & 1.0 \\
Bi-20 & hydrogel & 1.0 & 0.8 & 0.5 & 0.0 \\
& oleogel & 0.0 & 0.2 & 0.5 & 1.0 \\
Bi-30 & hydrogel & 1.0 & 0.7 & 0.5 & 0.0 \\
& oleogel & 0.0 & 0.3 & 0.5 & 1.0 \\
Bi-40 & hydrogel & 1.0 & 0.6 & 0.5 & 0.0 \\
& oleogel & 0.0 & 0.4 & 0.5 & 1.0 \\
\hline
\end{tabular}

Table III. Consistency indices $(\mathrm{K})$ and flow behaviour indices $(\mathrm{n}=3$, mean $\pm S D)$

\begin{tabular}{cccc}
\hline Formulation & $K$ & $n$ & $R^{2}$ \\
\hline Bi-10 & $291.55 \pm 3.76$ & $0.105 \pm 0.004$ & 0.77 \\
Bi-20 & $254.86 \pm 11.04$ & $0.138 \pm 0.005$ & 0.89 \\
Bi-30 & $203.33 \pm 7.81$ & $0.173 \pm 0.008$ & 0.96 \\
Bi-40 & $187.27 \pm 1.76$ & $0.2002 \pm 0.002$ & 0.98 \\
\hline
\end{tabular}

of both substances decreased when the concentration of oleogel increased (Bi-10 $>\mathrm{Bi}-20>$ Bi-30 > Bi-40) although the viscosity decreased. Typical release curves are shown in Fig. 3. It can be stated that oleogel slows the release from bigels and could be used to prolong the effect of the medicine (4). 
a)

60

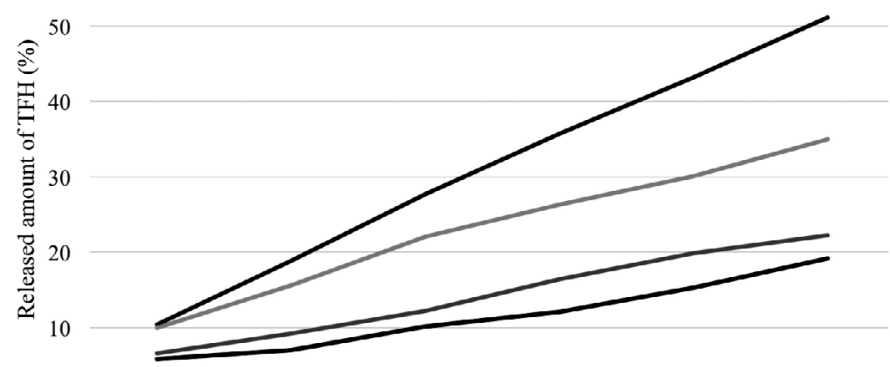

$1 \mathrm{~h}$ $\begin{array}{ccc}2 h & 3 h & 4 h\end{array}$

$6 \mathrm{~h}$

b) $\quad 60$

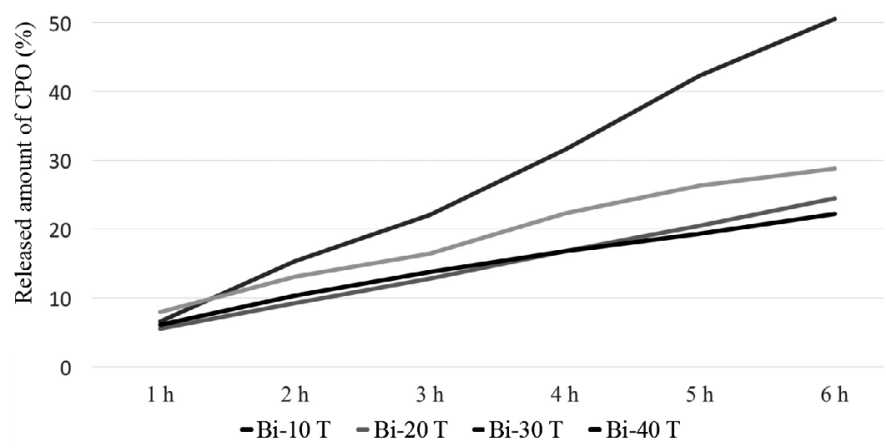

Fig. 3. Typical release curves of bigels of: a) terbinafine hydrochloride, and b) ciclopirox olamine. (Type B is shown in the graph - the amount of active substance is proportional to the amount of the phase) $(n=3)$.

Results of the release study can be associated with drug properties. TFH is a hydrophobic substance with $\log P 5.9$ (14), which slightly dissolves in water. Poloxamer 407 increases TFH solubility, though small solid particles might occur in hydrogel. The released amounts of TFH from Bi-10 were in the order of $\mathrm{B} \approx \mathrm{A}>\mathrm{C}>\mathrm{D}$ (Fig. 4a). Similar results were obtained in other formulations. CPO is sparingly soluble in water and is less lipophilic $(\log P 2.3(14))$ compared to TFH. The amount of CPO released in Bi-10 was in the following descending order: $C>B>D \approx A$ (Fig. $4 b$ ). Increased proportion of oleogel significantly reduced the released amount of active substances and the influence of insertion place became insignificant.

\section{Antifungal activity test}

The antifungal study was aimed at assessing whether the released amount of drug from the semi-solid form was able to induce a biological effect. Antifungal research re- 
a) 60

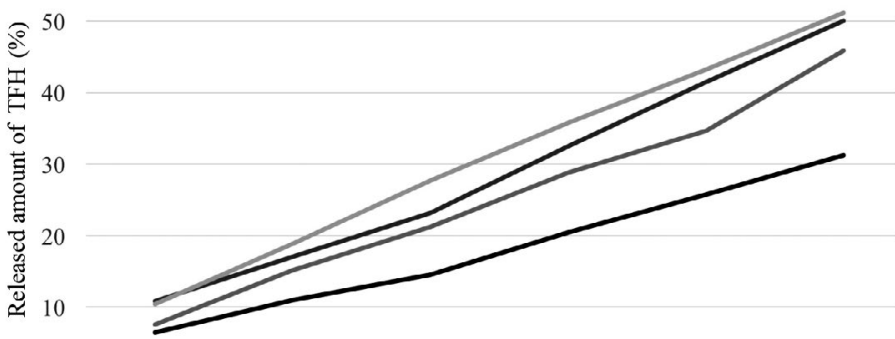

0
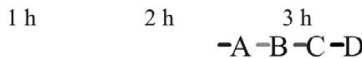

$4 \mathrm{~h}$

$5 \mathrm{~h}$

$6 \mathrm{~h}$

b)

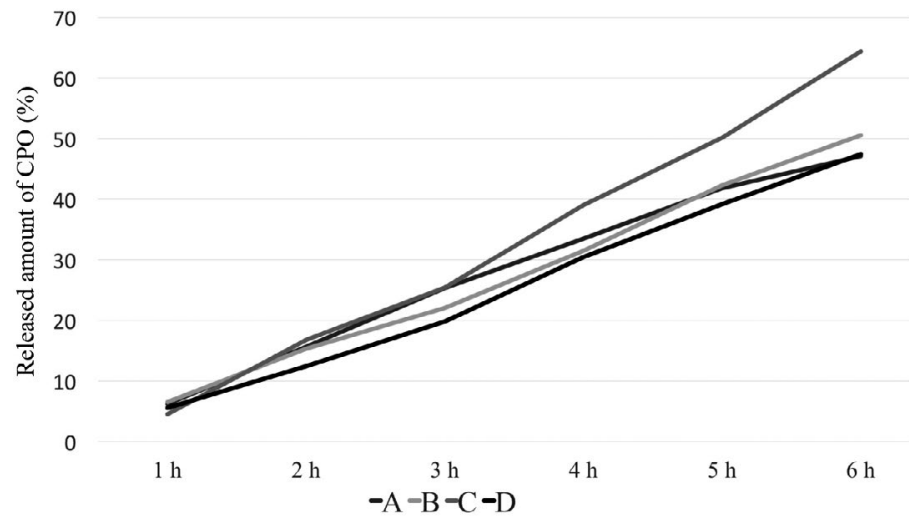

Fig. 4. Typical release curves of bigels of: a) terbinafine hydrochloride, and b) ciclopirox olamine (Bi-10 is shown in the graph) $(n=3)$. A - active substance in hydrogel, B - the amount of active substance in the phase is proportional to the amount of the phase in the formulation, $\mathrm{C}$ - equal amounts of active substance in both phases, D - active substance in oleogel.

vealed that bases without drug substances had no biological effect against $M$. canis. All formulations showed great inhibition of pathogenic fungi. The zones of inhibition were 69-71 $\mathrm{mm}$ for formulations of ciclopirox and more than $80 \mathrm{~mm}$ for terbinafine. The obtained results confirm the scientific literature data that a lower concentration of terbinafine hydrochloride is required to achieve a biological effect against dermatophytes compared to ciclopirox olamine (15). Tested bigels released the required amount of active substances, which ensured the effectiveness against $M$. canis.

\section{Stability study}

As the internal structure of the preparation may vary during storage, its rheological characteristics were evaluated. The structure became less stable when the consistency index decreased; there was even no visible phase separation. No changes were observed 
Table IV. Amount of terbinafine hydrochloride or ciclopirox olamine released in 6 hours $(\mathrm{n}=3$, mean \pm SD)

\begin{tabular}{|c|c|c|c|c|c|}
\hline Formulation & $\begin{array}{c}\text { Released } \\
\text { drug }\end{array}$ & A & B & $\mathrm{C}$ & $\mathrm{D}$ \\
\hline \multirow{2}{*}{ Bi-10 T } & $\%$ & $50.07 \pm 1.1$ & $51.14 \pm 5.36$ & $45.89 \pm 0.86$ & $31.20 \pm 1.81$ \\
\hline & $\mathrm{mg} / \mathrm{cm}^{2}$ & $3.76 \pm 0.08$ & $3.87 \pm 0.56$ & $3.41 \pm 0.42$ & $2.35 \pm 0.14$ \\
\hline \multirow[b]{2}{*}{$\mathrm{Bi}-20 \mathrm{~T}$} & $\%$ & $31.38 \pm 3.04$ & $34.97 \pm 3.52$ & $27.06 \pm 3.18$ & $20.79 \pm 0.29$ \\
\hline & $\mathrm{mg} / \mathrm{cm}^{2}$ & $2.36 \pm 0.22$ & $3.20 \pm 0.05$ & $2.12 \pm 0.02$ & $1.56 \pm 0.02$ \\
\hline \multirow{2}{*}{$\mathrm{Bi}-30 \mathrm{~T}$} & $\%$ & $21.03 \pm 2.15$ & $22.24 \pm 2.33$ & $23.51 \pm 0.29$ & $17.31 \pm 0.96$ \\
\hline & $\mathrm{mg} / \mathrm{cm}^{2}$ & $1.58 \pm 0.16$ & $1.73 \pm 0.13$ & $1.78 \pm 0.18$ & $1.30 \pm 0.07$ \\
\hline \multirow[b]{2}{*}{$\mathrm{Bi}-40 \mathrm{~T}$} & $\%$ & $20.21 \pm 1.45$ & $19.15 \pm 1.15$ & $21.90 \pm 3.57$ & $15.72 \pm 1.1$ \\
\hline & $\mathrm{mg} / \mathrm{cm}^{2}$ & $1.52 \pm 0.33$ & $1.47 \pm 0.16$ & $1.63 \pm 0.04$ & $1.18 \pm 0.08$ \\
\hline \multirow{2}{*}{ Bi-10 C } & $\%$ & $47.14 \pm 1.61$ & $50.59 \pm 1.65$ & $64.41 \pm 5.83$ & $47.43 \pm 1.03$ \\
\hline & $\mathrm{mg} / \mathrm{cm}^{2}$ & $3.54 \pm 0.12$ & $3.80 \pm 0.12$ & $7.84 \pm 0.51$ & $3.57 \pm 0.07$ \\
\hline \multirow{2}{*}{ Bi-20 C } & $\%$ & $26.65 \pm 0.64$ & $28.83 \pm 2.17$ & $35.61 \pm 2.97$ & $24.77 \pm 1.43$ \\
\hline & $\mathrm{mg} / \mathrm{cm}^{2}$ & $2.00 \pm 0.05$ & $2.19 \pm 0.16$ & $2.68 \pm 0.52$ & $1.86 \pm 0.11$ \\
\hline \multirow{2}{*}{$\mathrm{Bi}-30 \mathrm{C}$} & $\%$ & $17.81 \pm 1.76$ & $24.45 \pm 2.31$ & $26.44 \pm 1.65$ & $21.51 \pm 1.39$ \\
\hline & $\mathrm{mg} / \mathrm{cm}^{2}$ & $1.34 \pm 0.13$ & $1.85 \pm 0.05$ & $1.99 \pm 0.12$ & $1.62 \pm 0.10$ \\
\hline \multirow{2}{*}{ Bi- $40 \mathrm{C}$} & $\%$ & $15.10 \pm 0.84$ & $22.25 \pm 0.65$ & $21.90 \pm 1.56$ & $18.93 \pm 2.15$ \\
\hline & $\mathrm{mg} / \mathrm{cm}^{2}$ & $1.14 \pm 0.06$ & $1.65 \pm 0.13$ & $1.55 \pm 0.10$ & $1.42 \pm 0.16$ \\
\hline
\end{tabular}

in the organoleptic characteristics over 6 months at $25{ }^{\circ} \mathrm{C} / 60 \% \mathrm{RH}$ (Fig. 5). The results confirmed the data of scientific literature that bigels are stable at room temperature for 6-12 months (4).

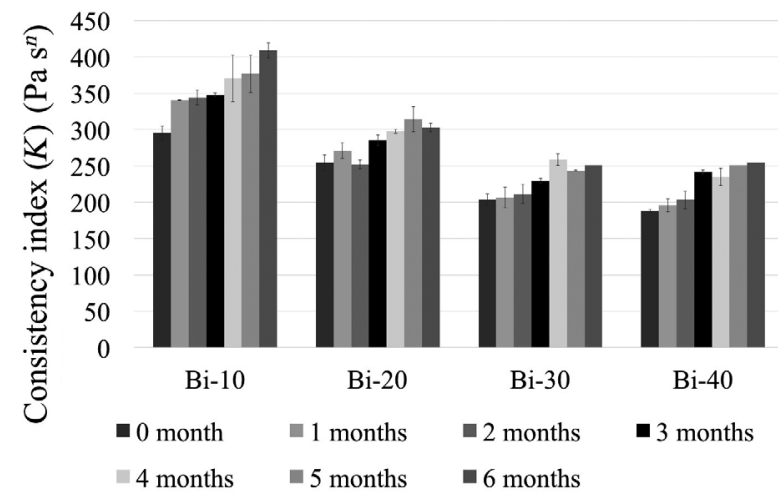

Fig. 5. Stability of blank bigels at $25^{\circ} \mathrm{C}(n=3$, mean \pm SD $)$. 


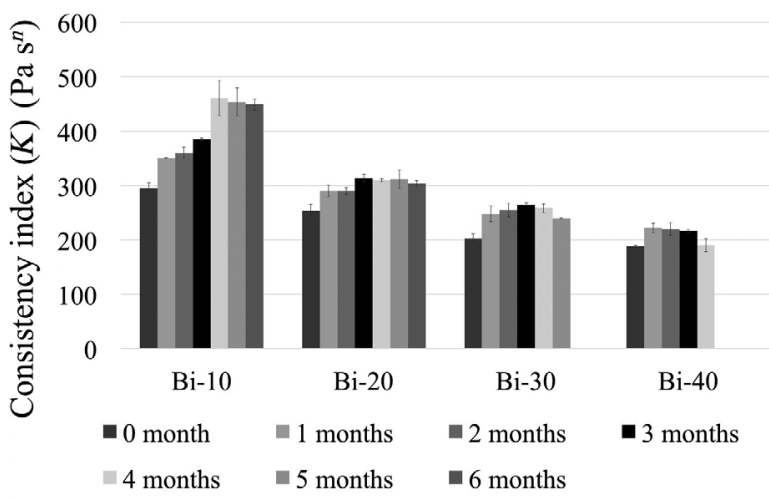

Fig. 6. Stability of blank bigels at $40^{\circ} \mathrm{C}(n=3$, mean \pm SD $)$.

Some changes were observed in the accelerated stability study. The consistency index started to drop and after a while phase separation was observed. Phase separation was observed after 4 months for Bi-40 and 5 months for Bi-30 at $40{ }^{\circ} \mathrm{C} / 75 \% \mathrm{RH}$ (Fig. 6). Lower viscosity and larger droplets could lead to lower stability of Bi-30 and Bi-40.

\section{CONCLUSIONS}

In the present study, bigels were successfully developed using the poloxamer 407 gel as a hydro phase and oleogel of liquid paraffin and polyethylene as an apolar phase. The microstructure of bigels showed the formation of emulsion droplets. Drug release and antifungal studies suggested that the tested bigels released a sufficient amount of ciclopirox olamine and terbinafine hydrochloride and were effective against Microsporus canis. The rheological test allowed assessing the changes of internal structure and determining the stability of the tested formulation. No changes in organoleptic properties were found over six months of storage and the structural change did not damage the formulations excessively. Results of the study show that the tested bigels are a promising form for antifungal substances and further comparative studies are needed.

\section{REFERENCES}

1. Y. C. Chen, D. Z. Liu, J. J. Liu, T. W. Chang, H. O. Ho and M. T. Sheu, Development of terbinafine solid lipid nanoparticles as a topical delivery system, Int. J. Nanomedicine 7 (2012) 4409-4418; https://doi.org/10.2147/IJN.S33682

2. I. F. Almeida, A. R. Fernandes, L. Fernandes, M. R. Pena Ferreira, P. C. Costa and M. F. Bahia, Moisturizing effect of oleogel/hydrogel mixtures, Pharm. Dev. Technol. 13 (2008) 487-494; https:// doi.org/10.1080/10837450802282447

3. S. Bangarwa, S. Garg and A. Aseri, A review on antifungal gels: as a topical drug delivery system, Int. J. Pharm. Technol. Biotechnol. 1 (2014) 48-55. 
4. V. K. Singh, I. Banerjee, T. Agarwal, K. Pramanik, M. K. Bhattacharya and K. Pal, Guar gum and sesame oil based novel bigels for controlled drug delivery, Colloids Surfaces B Biointerfaces 123 (2014) 582-592; https://doi.org/10.1016/j.colsurfb.2014.09.056

5. F. R. Lupi, L. Gentile, D. Gabriele, S. Mazzulla, N. Baldino and B. de Cindio, Olive oil and hyperthermal water bigels for cosmetic uses, J. Colloid Interface Sci. 459 (2015) 70-78; https://doi. org/10.1016/j.jcis.2015.08.013

6. M. J. Dolton, V. Perera, L. G. Pont and A. J. McLachlan, Terbinafine in combination with other antifungal agents for treatment of resistant or refractory mycoses: investigating optimal dosing regimens using a physiologically based pharmacokinetic model, Antimicrob. Agents Chemother. 58 (2014) 48-54; https://doi.org/10.1128/AAC.01466-13

7. M. K. Kathiravan, A. B. Salake, A. S. Chothe, P. B. Dudhe, R. P. Watode, M. S. Mukta, S. Gadhwe, The biology and chemistry of antifungal agents: A review, Bioorg. Med Chem. 20 (2012) 5678-5698; https://doi.org/10.1016/j.bmc.2012.04.045

8. K. Tabara, A. E. Szewczyk, W. Bienias, A. Wojciechowska, M. Pastuszka, M. Oszukowska, A. Kaszuba, Amorolfine vs. ciclopirox - Lacquers for the treatment of onychomycosis, Postep. Dermatol. Alergol. 32 (2015) 40-45; https://doi.org/10.5114/pdia.2014.40968

9. A. Mazurkeviciute, K. Ramanauskiene, M. Ivaskiene, A. Grigonis and V. Briedis, Modelling and biopharmaceutical evaluation of ciclopirox olamine gels, Acta Pol. Pharm. 74 (2017) 543-549.

10. J. Ma, Y. Lin, X. Chen, B. Zhao and J. Zhang, Flow behavior, thixotropy and dynamical viscoelasticity of sodium alginate aqueous solutions, Food Hydrocoll. 38 (2014) 119-128; https://doi. org/10.1016/j.foodhyd.2013.11.016

11. M. T. Islam, N. Rodriguez-Hornedo, S. Ciotti and C. Ackermann, Rheological characterization of topical carbomer gels neutralized to different pH, Pharm. Res. 21 (2004) 1192-1199; https://doi. org/10.1023/B:PHAM.0000033006.11619.07

12. M. M. Ibrahim, S. A. Hafez and M. M. Mahdy, Organogels, hydrogels and bigels as transdermal delivery systems for diltiazem HCL, Asian J. Pharm. Sci. 8 (2013) 46-54; https://doi.org/10.1016/j. ajps.2013.07.006

13. A. Kaur, S. Jaiswal and G. D. Gupta, Formulation and evalution of terbinafine hydrochloride topical gel, Indo. Am. J. Pharm. Res. 4 (2014) 2919-2927.

14. M. E. Herbig and D. H. Evers, Correlation of hydrotropic solubilization by urea with $\log$ D of drug molecules and utilization of this effect for topical formulations, Eur. J. Pharm. Biopharm. 85 (2013) 158-160; https://doi.org/10.1016/j.ejpb.2013.06.022

15. J. Leyden, Pharmacokinetics and pharmacology of terbinafine and itraconazole, J. Am. Acad. Dermatol. 38 (1998) 42-47; https://doi.org/10.1016/S0190-9622(98)70483-9 